\title{
Displacement sensing by piezoelectric transducers in high-speed lateral nanopositioning
}

\author{
Ali Bazaei, Member, IEEE, Mokrane Boudaoud, Massoud Hemmasian Ettefagh, Zhiyong Chen, Senior \\ Member, IEEE, Stephane Régnier
}

\begin{abstract}
The paper deals with the characterization of piezoelectric ceramic type sensors integrated in a compact high-speed 2-DOF nano-positioner to measure large signal stage displacement. Such transducers are very attractive because they provide measurements in a wide frequency bandwidth with low noise, they do not require bulky electronic modules and are cost effective compared to traditional sensors. The transducers are installed in series with piezoelectric actuators and thick ceramic insulators are incorporated between them to reduce the cross-talk. The sensor provides accurate position measurements as long as the nano-positioner displacement is within the sensing bandwidth. This is verified by large amplitude sinusoidal excitations of the nano-positioner along both $\mathrm{x}$ and $\mathrm{y}$ axes within the sensor bandwidth. The sensor can also measure the displacement in response to a single tone sinusoid at frequencies well above the dominant imaginary zeros of the interaction force, which is beyond the sensor bandwidth. Experimental measurements are compared with those obtained using a LASER interferometer sensor. The results allow obtaining precise calibration factors of the piezoelectric ceramic transducers with respect to the input frequencies and accurate displacement/voltage transfer characteristics for closed loop control purposes.
\end{abstract}

Index Terms-Displacement sensor, Piezoelectric transducer, Nanopositioner.

\section{INTRODUCTION}

High precision measurement of rapidly changing small displacements is required in many Micro and Nano positioning mechanisms such as nanopositioners for scanning probe microscopy [1]-[11], microscanner mirrors [12]-[14], and scanners in selective LASER sintering applications $[15]-[17]$.

A conventional method to measure displacement in flexure-based micro/nano-positioners is to use capacitive sensors [1]-[5], [18], [19]. Such sensors are generally expensive, need additional space and parts for physical installation, and require bulky electronic modules to generate a real-time signal proportional to the target displacement.

A. Bazaei, M. Boudaoud, and S. Régnier are with Sorbonne Université, Institut des Systèmes Intelligents et de Robotique, UMR 7222, ISIR, F-75005 Paris, France. M. H. Ettefagh is with the Department of Mechanical Engineering, Amirkabir University of Technology, Tehran, Iran. A. Bazaei and Z. Chen are with the School of Electrical Engineering and Computing, University of Newcastle Australia, Callaghan, NSW 2308. Emails: bazaei@isir.upmc.fr, ali.bazaei@newcastle.edu.au,_ mokrane.boudaoud@sorbonneuniversite.fr, hemmasian@aut.ac.ir, zhiyong.chen@newcastle.edu.au, stephane.regnier@sorbonne-universite.fr.
Other conventional displacement sensors, such as optical encoders, LASER interferometers, and Eddy current sensors also suffer from the foregoing issues [20]-[23].

Piezoresistive strain gauge elements have also been used to measure displacement in nanopositioners [18], [24], [25]. To do this, one normally needs to glue multiple strain gauges to the suspension beams, configure them in a Wheatstone bridge, and use DC power supplies and amplifiers to generate an output signal [6]. In addition, installation of conventional gauges is usually manual and can introduce undesirable asymmetry in the nanopositioner structure (especially for those with smaller dimensions), which is normally designed symmetrical (to reduce crosscoupling) and is fabricated by high precision automated machines. Automatic installation of piezoresistive strain gauges on flexure hinges is also possible by micromachining processes, however, it requires to fabricate the nanopositioner from fragile materials such as Silicon [7] or fabrication of the gauges on additional non-conductive flexible structures [26].

Piezoelectric sensors have been widely used to measure time-varying quantities such as changes in pressure, acceleration, temperature, force, and strain [13], [27]. Most piezoelectric actuators and sensors available in the market are lead zirconium titanate (PZT) ceramics [28], [29]. PZT ceramics have high sensitivity, a large range of operating temperature, inexpensive manufacturing cost, versatility in applications, and are chemically inert [30]. In contrast to polymer piezoelectric materials such as polyvinylidenefluoride (PVDF) [31], [32], PZT has high stiffness and high compressive strength to tolerate high force values in piezodriven nanopositioners [33], [34]. However, such sensors are not suitable for DC measurements and their sensitivity degrades as frequency approaches zero. In response to a constant mechanical load, a PZT sensor produces a limited amount of electric charge that gradually decays through limited input resistance of the circuit connected to the sensor [35]. Hence, such sensors are usually useful to detect variables that change sufficiently fast such as structural vibrations [28], [36], [37] and stage displacement in highspeed scanning devices, which is addressed in this paper. Fortunately, displacement sensors with DC measurement problems are still applicable in damping and tracking feedback control of nanopositioners [38]. The low-frequency issue of PZT sensors can also be resolved considerably by low-frequency equalizers or high input impedance elec- 


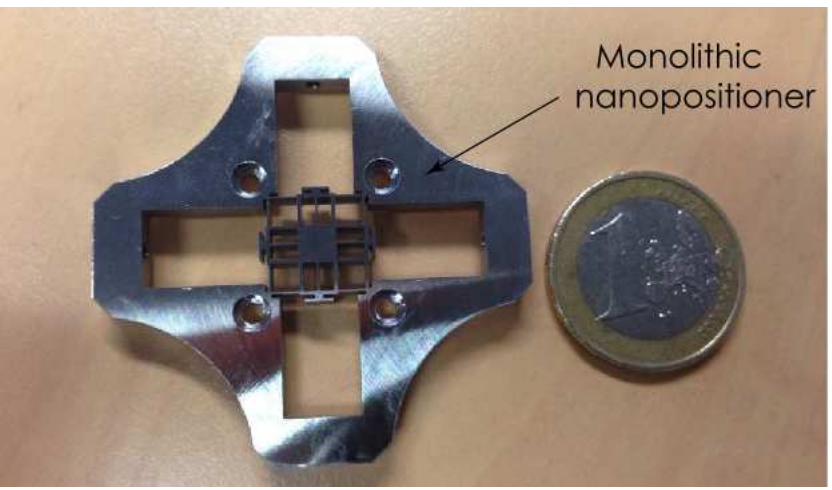

Fig. 1. Top-view of the $4 \mathrm{~mm}$ thick monolithic nano-positioner before the integration of the PZT actuators and sensors. The monolithic structure was fabricated from stainless-steel by EDM wire cutting.

tronic circuits [39]-[41]. PZT sensors can also exhibit high drift if driving high input impedance voltage amplifiers or charge amplifiers with finite input impedance [42]-[44]. On the other hand, their small noise power density at higher frequencies is very attractive compared to other kinds of sensors [23]. Moreover, they have small foot prints and do not require additional bulky parts for installation or complicated electronic instruments. They have also been used to detect underwater vibrating objects [45], force in tactile sensors [35], [46], [47], slip in prosthetic hands [48], and pressure in prosthetic limbs [49], [50]. However, their application as a direct displacement sensor is very limited, especially in Micro and Nano positioning mechanisms.

The electric charge induced by mechanical loading in piezoelectric materials can be used to detect high precision displacement of vibrating elements [51], [52]. However, such detection methods require fabrication of highly sensitive devices such as single-electron transistors (SET), which cannot properly operate at room temperature. In addition, the vibrating element has to be in contact with the SET island, which can limit the dynamic range of the sensor. In [8], [9], piezoceramic and strain gauge sensors have been combined by high-pass and low-pass filters to provide a low noise feedback signal for vibration damping in flexure-based micro/nano-positioners. However, neither the piezoceramic sensor nor the combined signals have been characterized for large signal measurement of the target displacement and only strain gauge and conventional sensors have been used to measure the nano-positioner displacement. In [53], piezoelectric patches have been used to measure the displacement of a target via the resonance frequency of a cantilever. In this method, the cantilever length has to vary with the target displacement and a sensor-actuator piezo patch pair excite the cantilever at its resonance frequency through an analog feedback circuit. In addition, only static characteristics of the sensor have been investigated in [53] and the sensing performances of the time-varying displacements, which is mainly affected by the feedback system, has not been addressed.

This paper addresses a comprehensive characterization (a)

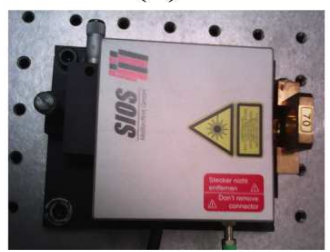

(c)

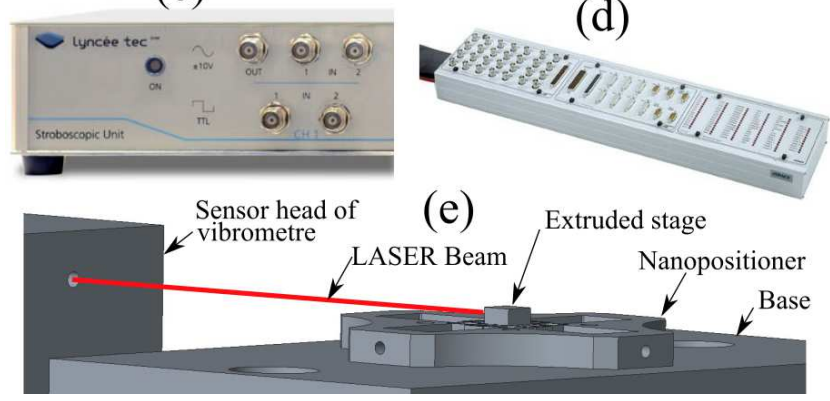

Fig. 2. Experimental setup: (a) Sensor Head of LASER Vibrometre, (b) Nanopositioner while laser beam hitting the extruded part of in the middle stage, (c) LynceeTec Stroboscopic unit (d) dSPACE I/O panel (e) Schematic of stage displacement measurement mechanism by the LASER vibrometre.

of piezoelectric ceramics in sensing time-varying and largesignal displacement of a piezo-driven flexure-based nanopositioning stage (Fig. 1). Compared to the methods mentioned in [8], [9], [51]-[53], here, the induced voltage in the PZT sensor is directly used for displacement measurement without being combined by an auxiliary sensor or keeping an additional electromechanical system at its resonance frequency. In addition, the proposed method does not rely on additional sensitive or complicated devices. Moreover, the target structure is not in direct contact with the sensor structure so that large displacement of the target does not interrupt the sensor operation. Hence, the proposed sensor does not suffer from a very limited dynamic range. The proposed displacement sensing and calibration approach is applicable to almost any piezo-driven nanopositioner and also to larger structures with commercial piezoelectric sensors [36]. In the approach considered here, the PZT sensor is located in an almost stationary location where the sensor response is directly affected by the interaction force rather than the stage displacement. This configuration not only increases the dynamic range of the displacement sensing method but also minimizes effect of the sensor body on the dynamic response of the stage.

The paper is structured as follows. Section II describes the experimental setup. The frequency analysis and the large signal characterization of the sensor are presented in sections III and IV respectively. The sensor characterization beyond the bandwidth is the treated in section V. The analysis of the noise and the resolution of the measurement is presented in VI. The paper is concluded in section VII. 


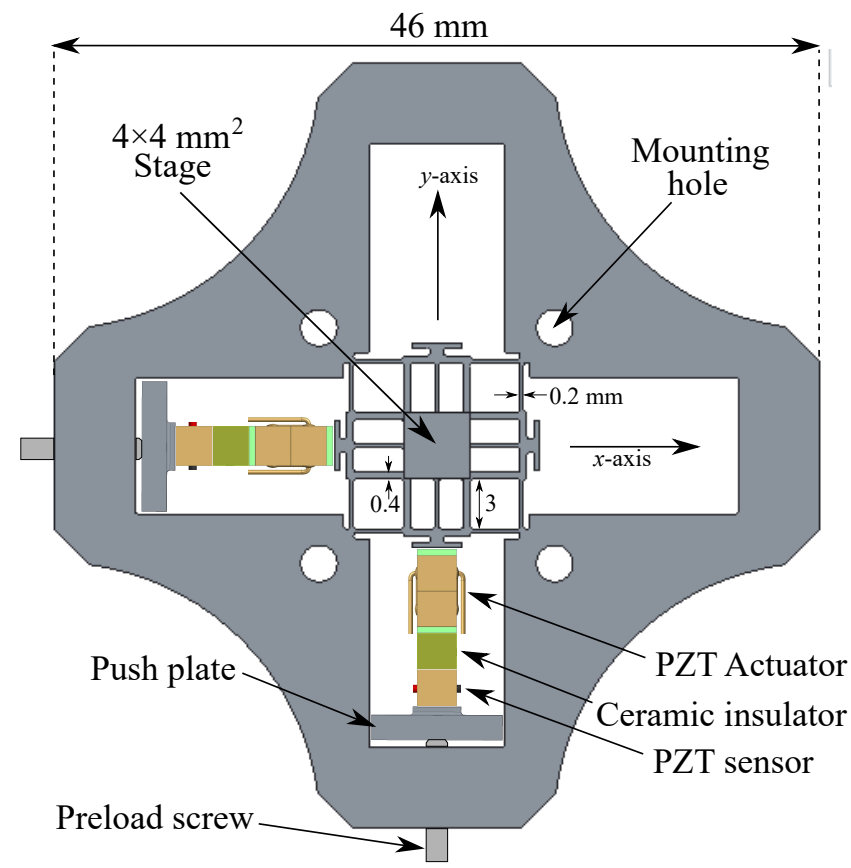

Fig. 3. Top-view schematic diagram of the nanopositioner and the PZT sensor-actuator pair of each axis with additional parts for insulation and installation.

\section{EXPERIMENTAL SETUP}

Figure 2 shows different parts of the experimental setup. A more exact architecture of the monolithic flexure-based $x-y$ nano-positioner integrating the PZT actuators and sensors is also shown in Fig. 3. The nano-positioner is symmetric with respect to the $x$ and $y$ axes. The square stage in the middle can be driven along $x$ and/or $y$ axes by PZT stack actuators via slender suspension beams. It is extruded about $2 \mathrm{~mm}$ to provide a valid measurement of the stage displacement by a LASER interferometer, as shown in Fig. 2 (e). For each axis, a piezoceramic is installed as a PZT sensor in series with the actuator to measure the stage displacement along that axis. To reduce cross-talk from the actuator to the sensor, an inactive ceramic insulator is included between each PZT sensoractuator pair. The insulator thickness here is much larger than those used in [8], [9]. With this arrangement, a better measurement of the displacement is expected from the PZT sensor especially at large signal displacements where the actuator is driven by high voltage signals. The dimensions of the ceramic insulator are similar to those of the PZT sensor. The PZT sensor is a commercial piezo chip actuator whose specifications are reported in Table I along with those of the PZT stack actuator. The three ceramic parts of each axis have the same cross-sectional area of $2.5 \times 2.5 \mathrm{~mm}^{2}$. They are hold together by a preload screw and a push plate after careful alignments without applying any adhesive which can sacrifice displacement range due to insufficient rigidity. To assist in this alignment during the assembly, an additional fixture whose top surface has different heights was fabricated, which is inserted in corresponding ditches of a base part designed for the nanopositioner, as shown in Fig. 4. For each axis, we experimentally maximized the range of stage displacement by tuning the preload force via the preload screw, while applying a large signal $240 \mathrm{~Hz}$ single tone sinusoidal actuation on the PZT actuator and measuring the stage displacement by the LASER interferometer.
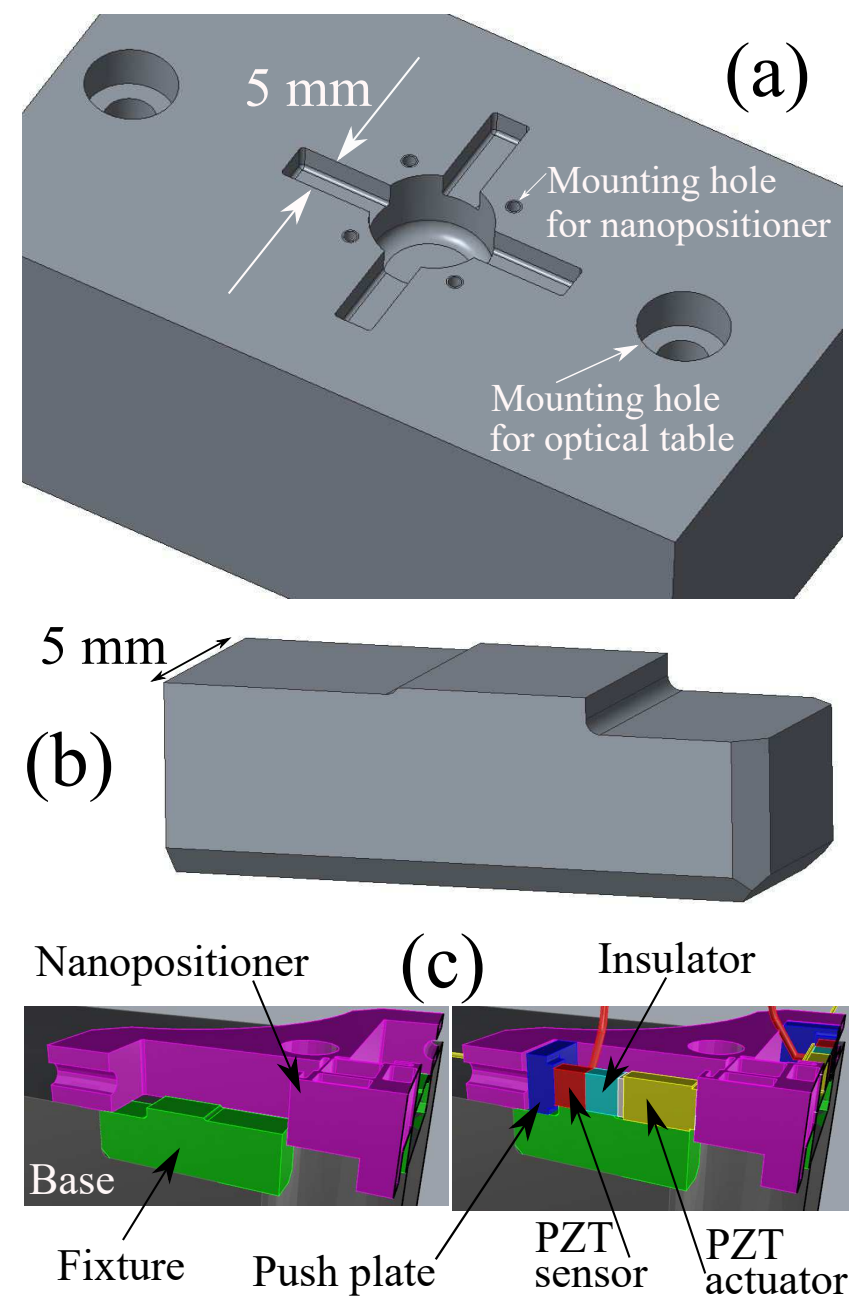

Fig. 4. (a) The Base for installation of the nanopositioner on an optical table after assembling the sensors and actuators. The $5 \mathrm{~mm}$ wide ditches in the base are to insert the fixture shown in (b) for vertical alignment of the three ceramic parts during the assembly process, as indicated in (c). The fixture is removed after the assembly to avoid any dry friction during normal operation and provide room for wiring.

For each axis, a voltage amplifier is used with a gain of 20 to drive the PZT actuator. For the frequency response measurement, the amplifier input is a biased sinusoid so that the voltage applied to the PZT actuator remains within the safe drive range of $0-150 \mathrm{~V}$. With a bias voltage of $70 \mathrm{~V}$ on the PZT actuator, Fig. 5 shows the experimental frequency response of each individual axis in the range of $50 \mathrm{~Hz}-30 \mathrm{kHz}$. The transfer considered here is from the amplifier input signal to the stage displacement in $\mu \mathrm{m}$ measured by the LASER sensor. The dominant resonance modes along $x$ and $y$ axes are around $15.95 \mathrm{kHz}$ and $17.15 \mathrm{kHz}$, respectively, where there is a $90^{\circ}$-phase-lag for 

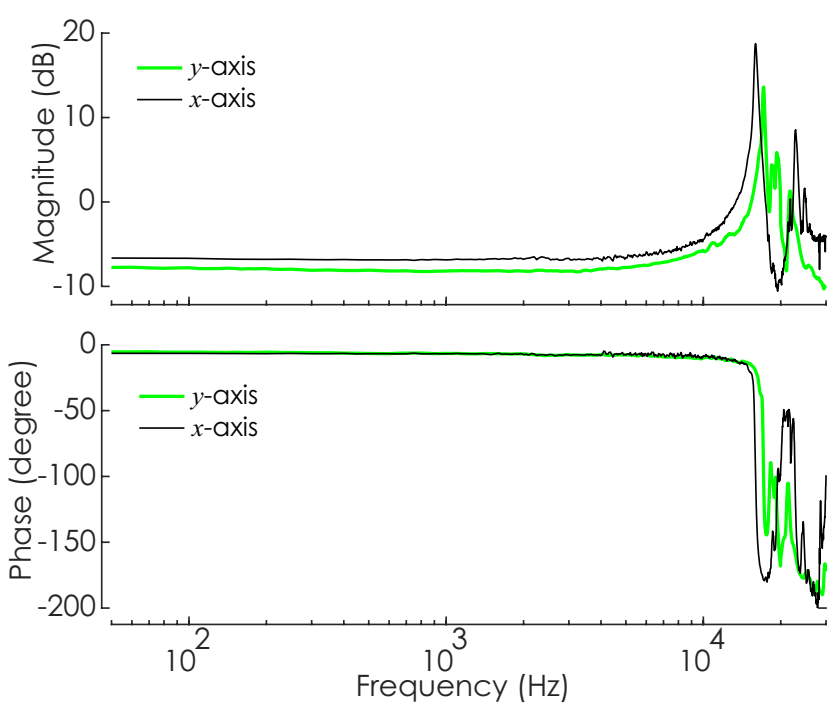

Fig. 5. Frequency responses of the nanopositioner from the amplifier input to the stage displacement measured by LASER interferometer along each individual axis of the nanopositioner.

TABLE I

SPECIFICATIONS OF THE PIEZOELECTRIC STACK AND CHIP ACTUATORS used AS PZT aCtuator AND SEnSOR, RESPECTIVEly (Models No. PK4DLP2 AND PA4DGW in [54]).

\begin{tabular}{lcc}
\hline Physical Property & PZT Stack & PZT Chip \\
\hline Drive Voltage Range & $0-150 \mathrm{~V}$ & $0-150 \mathrm{~V}$ \\
Free Stroke at 150 V & $5.2 \mu \mathrm{m} \pm 15 \%$ & $2.3 \mu \mathrm{m} \pm 15 \%$ \\
Recommended Preload & $100 \mathrm{~N}$ & $100 \mathrm{~N}$ \\
Blocking Force at 150 V & $250 \mathrm{~N}$ & $250 \mathrm{~N}$ \\
Dimensions in mm & $2.5 \times 2.5 \times 5.0$ & $2.5 \times 2.5 \times 2.3$ \\
Electrical Capacitance & $100 \mathrm{nF} \pm 15 \%$ & $50 \mathrm{nF} \pm 15 \%$ \\
Resonance Frequency & $250 \mathrm{kHz}$ & $650 \mathrm{kHz}$ \\
Operating Temperature & -25 to $130^{\circ} \mathrm{C}$ & -25 to $130^{\circ} \mathrm{C}$ \\
Curie Temperature & $230^{\circ} \mathrm{C}$ & $230{ }^{\circ} \mathrm{C}$ \\
\hline
\end{tabular}

both axes. The difference between the low frequency levels can be attributed to the tolerances of the PZT actuator specifications and different preload force values of $\mathrm{x}$ and $\mathrm{y}$ axes.

\section{Frequency Responses of PZT Sensors}

To characterize the PZT sensors, it is beneficial to identify the frequency range in which they can provide valid measurements of the stage displacement. Using the foregoing sinusoidal actuation settings, the experimental frequency response data of Fig. 6 is obtained in the range of $1 \mathrm{~Hz}-30 \mathrm{kHz}$ for each axis. The transfer function is from the amplifier input to the PZT sensor voltage of the driven axis.

The dominant resonance modes detected by the PZT sensors are around $16.1 \mathrm{kHz}$ and $17.5 \mathrm{kHz}$, respectively. The values and order of these frequencies for $x$ and $y$ axes are almost consistent with those obtained by the LASER interferometer for the stage displacement. However, there is a $90^{\circ}$-phase-lead at the dominant modes for the PZT sensors of both axes.

Each PZT sensor exhibits a pair of lightly damped
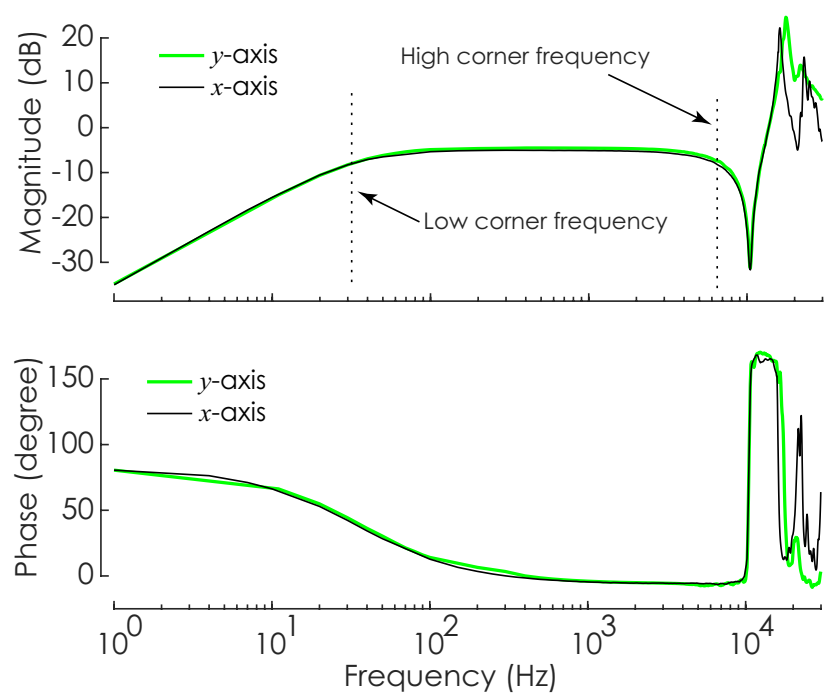

Fig. 6. Frequency responses of the nanopositioner from the amplifier input to the PZT sensor along each individual axis of the nanopositioner.

dominant imaginary zeros, which are not observed in the bode plots of the stage displacement in Fig. 5. The dominant zero pairs for both axes are located at almost identical frequency points of $10.5 \mathrm{kHz}$. A modal analysis of the nanopositioner structure reveals that the unloaded nanopositioner has a natural dominant resonant mode along the horizontal axes, as depicted in Fig. 7, at almost the same frequency of the zero pair. At this actuation frequency, the interaction force between the PZT actuator and the stage is considerably reduced as the stage requires nearly zero external force to vibrate at its unloaded natural mode. Since the PZT sensor primarily responses to the interaction force, it exhibits such dominant imaginary zeros that does not appear in the actual stage displacement. This fact can also be proven by a mass-spring model as presented in [8]. We can now justify why the frequency values at the zeros for $\mathrm{x}$ and $y$ axes are almost identical whilst those at the actual dominant resonance modes differ slightly. The actual resonance mode is determined by the physical specifications of both the monolithic nanopositioner and the ceramics while the zero is only determined by the specifications of the stage and the suspension beams. Some physical specifications for the PZT ceramics such as free-stroke and stiffness have tolerance values larger than $10 \%$, while the EDM (Electrical Discharge Machining) fabrication tolerance for the monolithic structure is much smaller. Hence, the monolithic structure is almost identical along $\mathrm{x}$ and $\mathrm{y}$ axes and so are the actual zero locations along them.

As the actuation frequency decreases towards zero, the magnitude response of the PZT sensors decrease by $20 \mathrm{~dB}$ per decade with a phase lead that tends to $90^{\circ}$. This indicates a zero at the origin, which is consistent with the response of a first-order high-pass filter made by series combination of a capacitor and a resistor. The total 


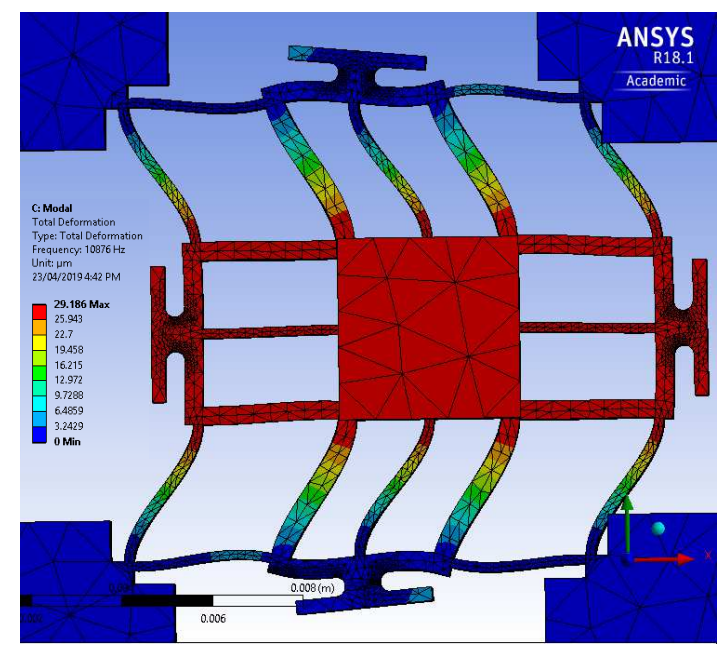

Fig. 7. Magnified mode shape of the monolithic structure, indicating the total deformation pattern along $x$-axis during the first free vibration mode at natural frequency of $10.88 \mathrm{kHz}$, whilst the mounting screw holes are fixed and no PZT ceramic exists in the nanopositioner. The data was predicted by a finite-element modal analysis.

resistance of instruments driven by a PZT sensor during the experiment was measured to be $R=88 \mathrm{k} \Omega$. The measured capacitance of the PZT sensors after preloading is around $C=60 \mathrm{nF}$. Hence, the $3-\mathrm{dB}$ corner frequency of the high-pass filter is $(2 \pi R C)^{-1}=30.14 \mathrm{~Hz}$, which is very close to the measured low corner frequencies in Fig. 6 around $32 \mathrm{~Hz}$. The high 3-dB corner frequency of the PZT sensors due to the dominant imaginary zeros is around $6400 \mathrm{~Hz}$. Hence, the frequency range in which the sensor can be used for direct measurement of stage displacement is $[32,6400] \mathrm{Hz}$. Note that the low corner frequency can further be reduced if the PZT sensor is connected to a load with higher input resistance.

\section{Large Signal Characterization}

A Sinusoidal waveform is the best profile for large signal characterization of lightly damped resonant systems, because it has almost no frequency component out of the frequency range of interest. To characterize the PZT sensor of y-axis, a $500 \mathrm{~Hz}$ sinusoidal voltage plus a DC bias is applied to the amplifier input, which swings between zero and $6.93 \mathrm{~V}$. This frequency is well within the frequency range of the PZT sensor for a direct displacement measurement. After simultaneously recording the signals from the LASER interferometer corresponding to the stage displacement, the PZT sensor, and the amplifier input, the PZT and the input signals have been scaled to have almost the same swing levels as the stage displacement. To scale each signal, it has been multiplied by a constant coefficient and then augmented by another constant.

Fig. 8 (a) shows the resulting signals after the scaling in the time domain. The scaling coefficients and constants are also included in Table II. In this experiment, the PZT sensor and the stage displacement signals almost overlap in the time domain. This indicates that the PZT sensor signal

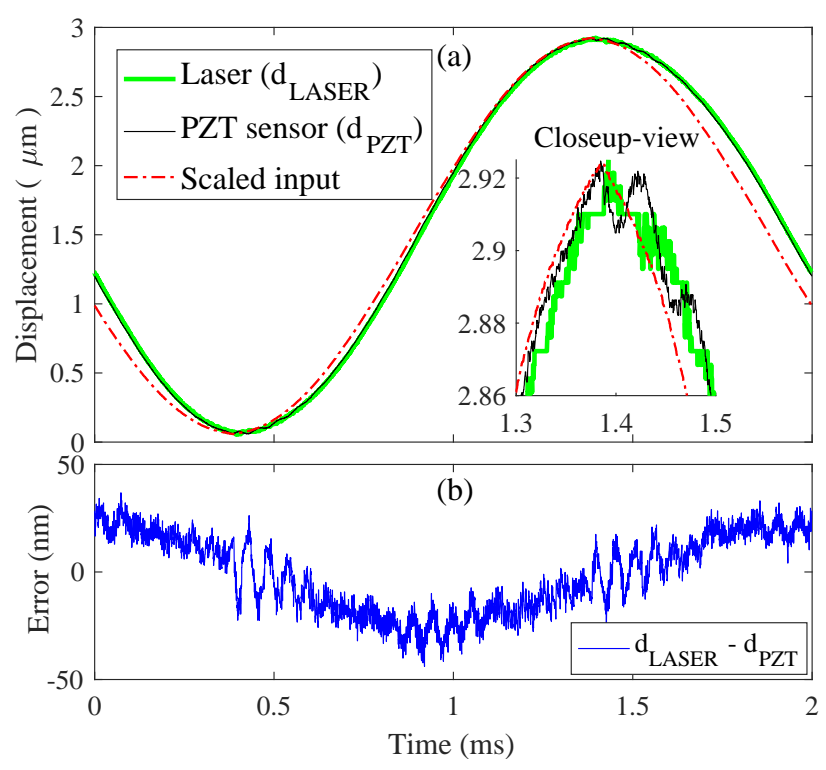

Fig. 8. Experimental response of the PZT sensor to large signal sinusoidal excitation of $\mathrm{y}$-axis at $500 \mathrm{~Hz}$.

TABLE II

CoEfFicients And CONSTANTS USED TO SCALE The PZT SENSOR AND AMPLIFIER INPUT SIGNALS.

\begin{tabular}{lcc}
\hline Signal & coefficient $\left(\frac{\mu m}{\mathrm{~V}}\right)$ & constant $(\mu m)$ \\
\hline PZT-sensor (y-axis) & 0.726 & 1.54 \\
Input voltage (y-axis) & 0.412 & 0.062 \\
PZT-sensor (x-axis) & 0.803 & 0.193 \\
Input voltage (x-axis) & 0.452 & 0.135 \\
\hline
\end{tabular}

can provide valid measurement of the stage displacement with a sensitivity of $1.377 \frac{\mathrm{V}}{\mu m}$ along y-axis (inverse of the scaling coefficient), if the power spectrum of the excitation is well within the sensor frequency range.

Figure 8 (b) shows the displacement measurement error, which is the difference between the actual displacement and the PZT sensor measurement. Small vibrations are observed around $18 \mathrm{kHz}$ in the error, which is close to the resonance frequency of $y$-axis. Such vibrations are excited by the deviation of the actuation signal from an ideal sinusoidal profile, as indicated by the sharp peak in the closeup-view of Fig. 8 (a). There is also a timelead of $t_{l}=6 \mu \mathrm{s}$ for the PZT sensor with respect to the displacement. This alternative source of error is due to the foregoing high-pass property of the sensor. The timelead can be used to calculate the low corner frequency of the PZT sensor at $f_{L} \cong 2 \pi t_{l} f^{2}=9.4 \mathrm{~Hz}$ from the firstorder high-pass model at $f=500 \mathrm{~Hz}$. In this experiment, the total load connected to the PZT sensor has a higher impedance than the load during the frequency response measurement, leading to a smaller value for the low corner frequency.

Fig. 9 shows the responses of the LASER and the PZT sensors versus the scaled input during the foregoing large signal sinusoidal excitation. These nonlinear contours, which originate from PZT actuator hysteresis, indicate 


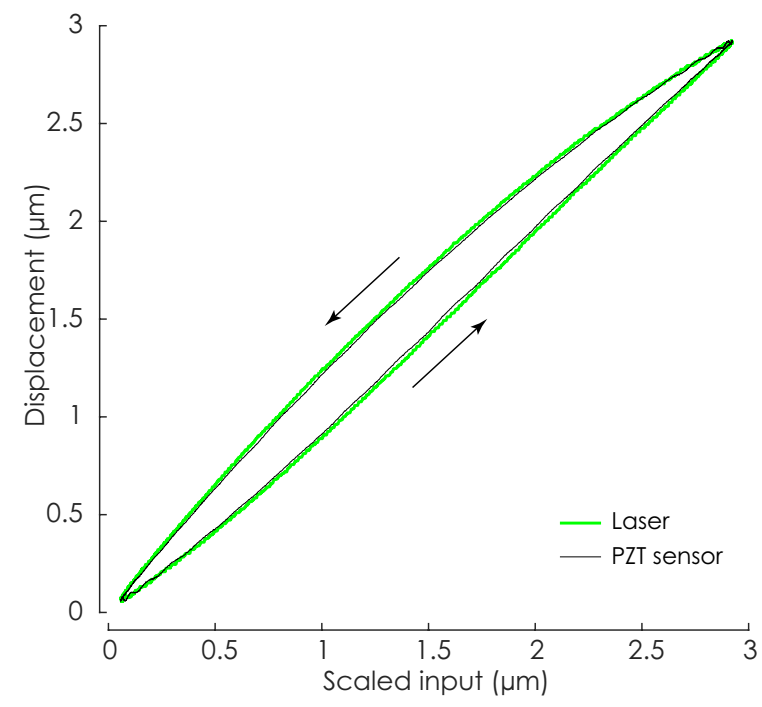

Fig. 9. Hysteresis curves of stage displacement measured by LASER interferometer and PZT sensor by the $500 \mathrm{~Hz}$ sinusoidal actuation.
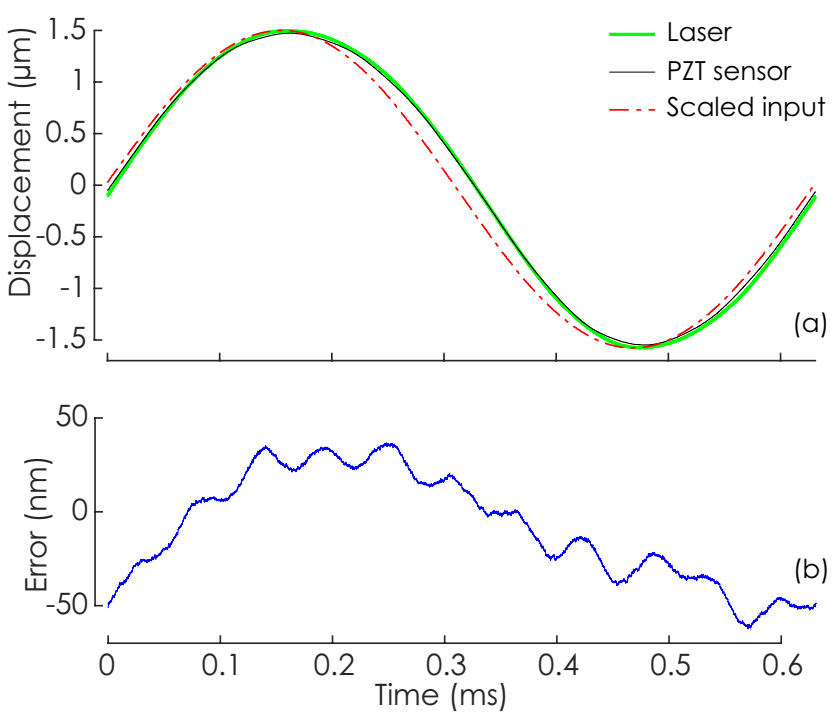

Fig. 10. Response of the PZT sensor to large signal sinusoidal excitation of $\mathrm{y}$-axis at $1583 \mathrm{~Hz}$.

that the PZT sensor can precisely follow large deviations of the stage displacement from an ideal sinusoidal profile.

As shown in Fig. 10, the sensing performance of the PZT sensor of $y$-axis has been also evaluated at a different excitation frequency of $1583 \mathrm{~Hz}$, whilst using the scaling coefficient of $0.726 \frac{\mu m}{\mathrm{~V}}$ obtained from the $500 \mathrm{~Hz}$ sinusoidal test. This scaling coefficient was also used to examine the PZT sensor at more different frequency values within the sensing bandwidth. Figure 11 summarizes large signal peak-to-peak displacement of the stage and the resulting range of measured values by the PZT sensor in each period. The average value of the absolute error in each test is also reported in Table III. As frequency was increased beyond $1583 \mathrm{~Hz}$, we had to reduce the excitation amplitude to prevent distortion and keep the voltage profile at the amplifier output almost sinusoidally.

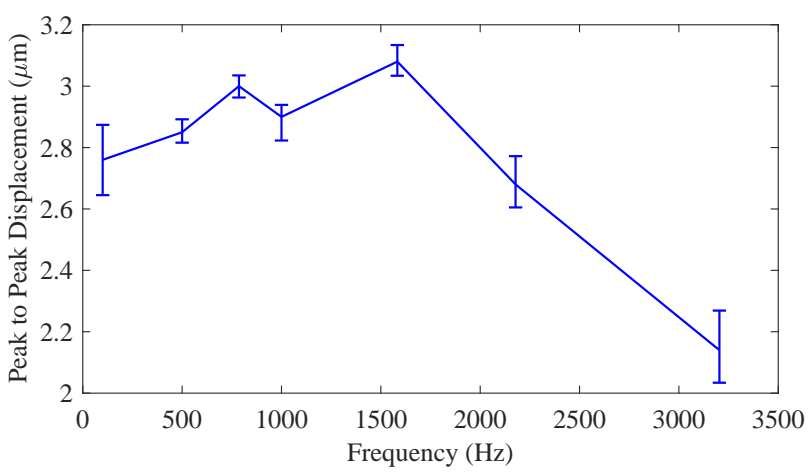

Fig. 11. Evaluation of PZT sensor performance by large signal sinusoidal excitation of $y$-axis at different frequency values, using a constant calibration coefficient within the sensing bandwidth. The vertical axis represents the peak-to-peak displacement of the stage. The ranges of PZT sensor measurement are indicated by error bars.

TABLE III

ACCURACY OF THE PZT SENSOR IN PREDiCtion OF Y-AXIS STAGE DISPLACEMENT IN EACH SINUSOIDAL TEST REPORTED IN FIG. 11.

\begin{tabular}{lccccccc}
\hline Freq.(Hz) & 100 & 500 & 786 & 1000 & 1583 & 2178 & 3204 \\
\hline Error $(\mathrm{nm})$ & 68.1 & 17.8 & 19.3 & 26.9 & 25.7 & 48 & 70 \\
\hline
\end{tabular}

The distortion is mainly attributed to current limitations of the amplifier and the PZT actuator. The range of PZT measurement error can also increase with frequency as imperfect sinusoidal actuation and hysteresis nonlinearity can create undesirable high order components close to the dominant resonance modes, which are far beyond the PZT sensor bandwidth. For example, the frequency value of the minor vibrations in the error profile of Fig. $10(\mathrm{~b}))$ is around $18 \mathrm{kHz}$, which is close to the dominant resonance frequency of $\mathrm{y}$-axis and far beyond the high corner frequency of the PZT sensor at $6400 \mathrm{~Hz}$. The measurement error also increases as frequency is reduced toward the low corner frequency due to the increase of the time-lead in PZT sensor response.

Figure 12 illustrates the performance of the PZT sensor with the same scaling coefficient as before in response to a non-sinusoidal excitation waveform. The actuation profile is continuous with a fundamental frequency well within the PZT sensing bandwidth. However, such triangular waveforms have strong odd harmonics that can excite the dominant resonance mode of the nanopositioner, considerably. The vibrations induced in the PZT sensor voltage and the actual stage displacement (LASER) at the resonance mode are out of phase, as shown in the closeup view of Fig. 12 (a). Hence, their contributions in the error profile of the PZT sensor add up, as shown by the high-frequency vibrations in Fig. 12 (b). Ignoring the small out-of-bandwidth vibrations in Fig. 12 (a), the PZT sensor closely follows large signal displacement of the stage and the limits of the sensing error in Fig. 12 (b) would be reduced, considerably.

Fig. 13 shows the experimental responses of the $x$-axis of the nanopositioner to a large signal sinusoidal excitation of 

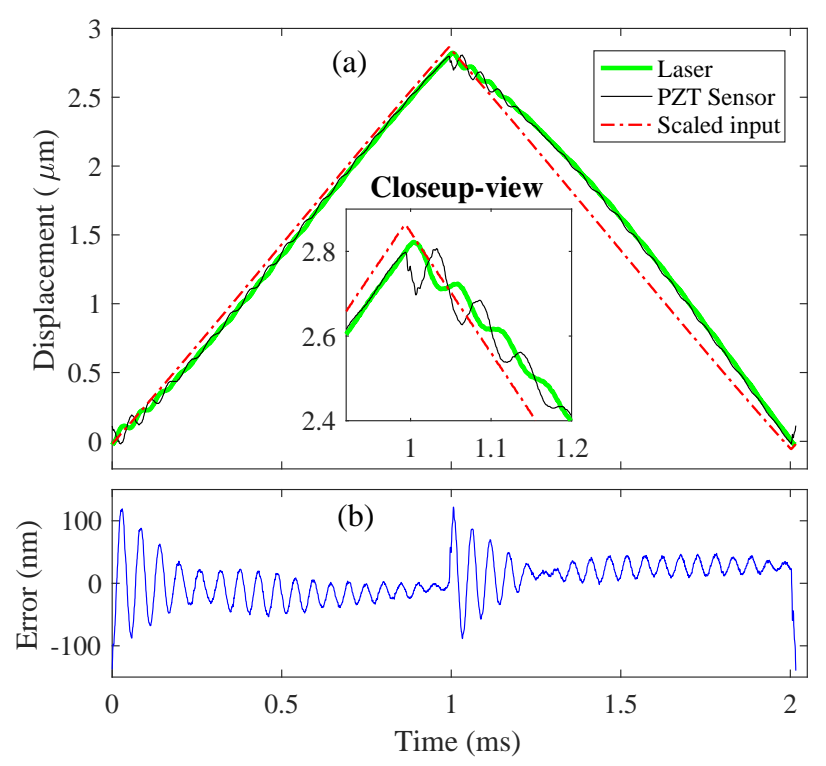

Fig. 12. Evaluation of the PZT sensor performance by a large signal triangular excitation of $y$-axis actuator with fundamental frequency of $496 \mathrm{~Hz}$ and peak-to-peak voltage of $137 \mathrm{~V}$.
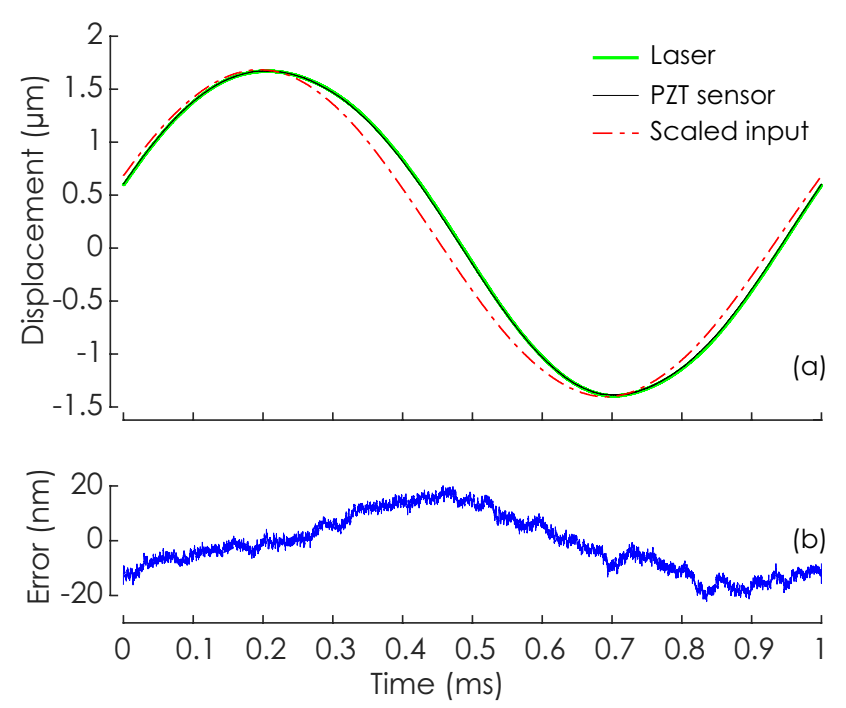

Fig. 13. Experimental response of the PZT sensor to large signal sinusoidal excitation of $\mathrm{x}$-axis at $1 \mathrm{kHz}$.

the $\mathrm{x}$-axis at $1 \mathrm{kHz}$, where the PZT sensor and the input signals have been scaled and biased as before to match the stage displacement levels with the coefficients and the constants reported in Table II for the $x$-axis. The PZT sensor signal has a sensitivity around $1.245 \frac{\mathrm{V}}{\mu m}$ and follows the time-varying stage displacement, accurately.

Using this sensitivity value, the stage displacement along $\mathrm{x}$-axis has been also measured by the PZT and the LASER sensors at different frequency values of $240 \mathrm{~Hz}$ and $2.5 \mathrm{kHz}$, as shown in Figures 14 and 15 . In the $240 \mathrm{~Hz}$ test, the PZT sensor leads the stage displacement by almost $17 \mu \mathrm{s}$, as indicated by the closeup view in Fig. 14 (a). Hence, the resulting low corner frequency of the $x$-axis PZT sensor is around $6.15 \mathrm{~Hz}$. Note that the PZT
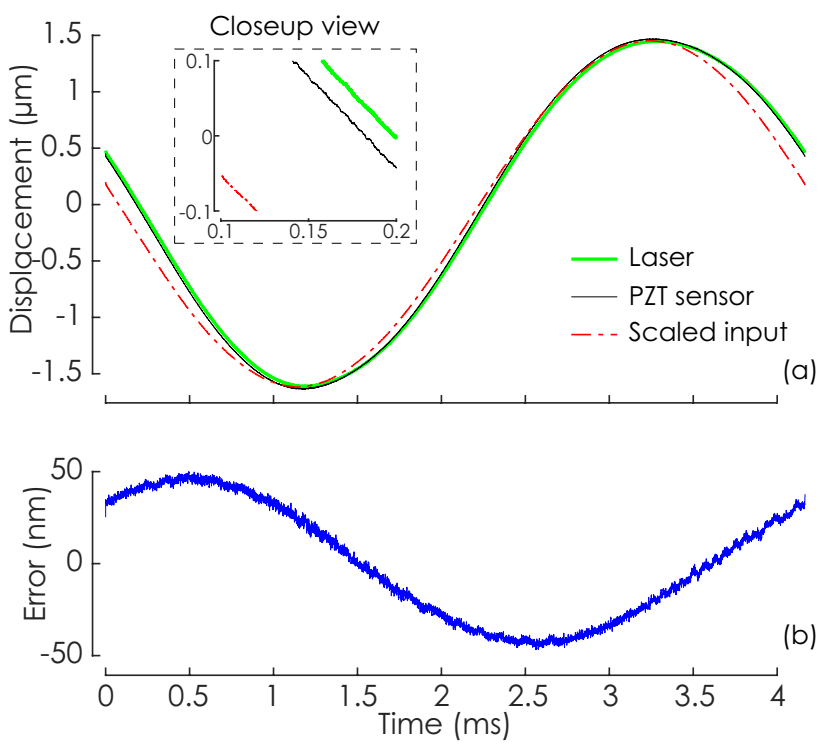

Fig. 14. Experimental response of the PZT sensor to large signal sinusoidal excitation of $\mathrm{x}$-axis at $240 \mathrm{~Hz}$.
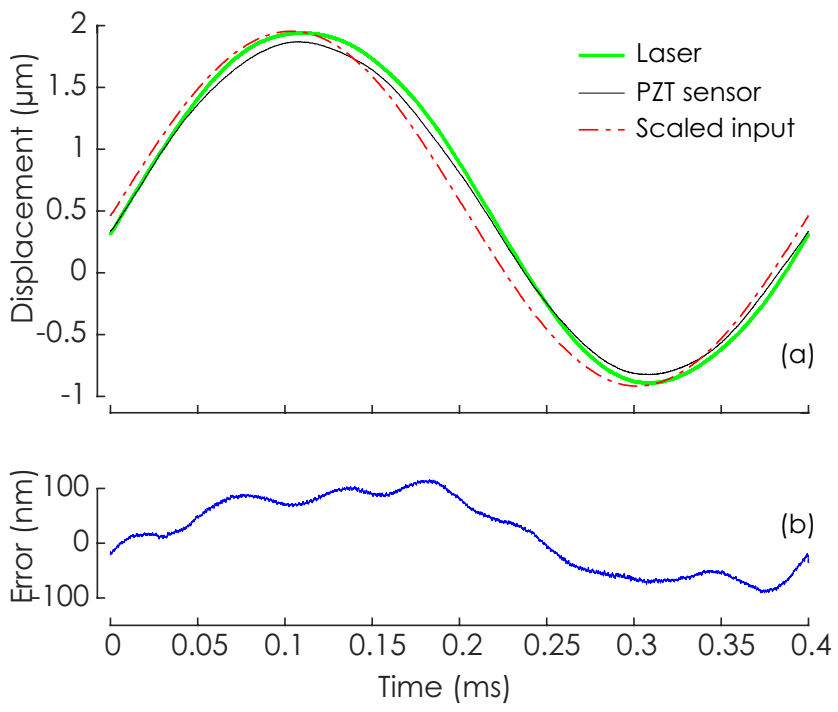

Fig. 15. Experimental response of the PZT sensor to large signal sinusoidal excitation of $\mathrm{x}$-axis at $2.5 \mathrm{kHz}$.

sensor still follows the stage displacement at $240 \mathrm{~Hz}$ with high accuracy. At the $2.5 \mathrm{kHz}$ test, there are vibrations around $18 \mathrm{kHz}$, which are visible in the error profile and degrade the accuracy of the displacement measurement. Such vibrations originate from the sharp resonance modes of the x-axis at $16 \mathrm{kHz}$ and $23 \mathrm{kHz}$, which are excited by high frequency components from the nonlinearity of the PZT actuator and the deviation of the actuation signal from an ideal sinusoid.

Overall, the acceptable overlaps between the LASER and the PZT responses indicate that the sensitivity of each PZT sensor is almost invariant over the frequency range mentioned in section III. 


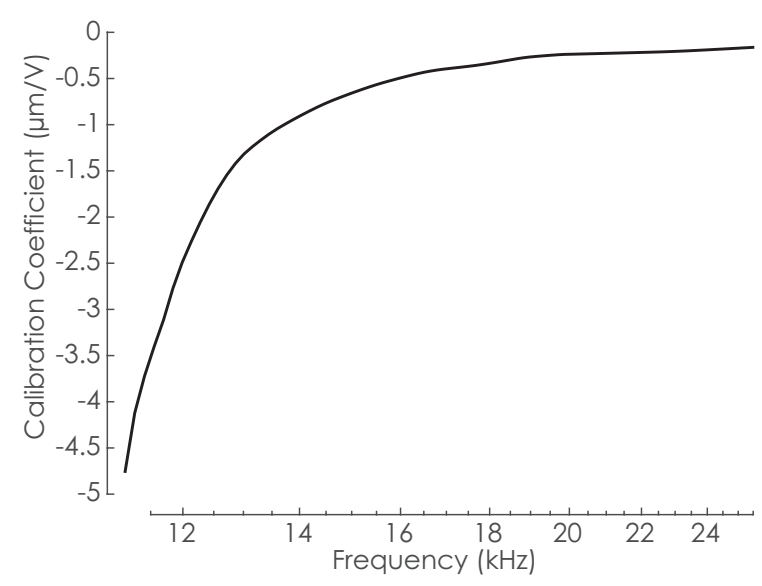

Fig. 16. Calibration coefficient of the $y$-axis PZT sensor for large signal sinusoidal excitation at the frequency range beyond the imaginary zeros.

\section{Sensor Characterization beyond the BANDWIDTH}

The frequency responses of the PZT sensors in Fig. 6 indicate a sharp increase of phase from 0 to $180^{\circ}$ around $10.5 \mathrm{kHz}$ due to the imaginary zeros, which does not happen for the displacement frequency responses in Fig. 5. Despite the displacement response, the PZT sensor response at frequency values well above the zero frequency (more than $13 \mathrm{kHz}$ ) is stronger than its response in the sensing bandwidth. Hence, it is valuable to characterize the displacement sensing performance of the sensor beyond the sensing bandwidth.

A number of large-signal single tone sinusoidal excitations have been carried out as before at a number of frequencies above the frequency of the imaginary zeros for the $y$-axis. The displacement and the PZT sensor
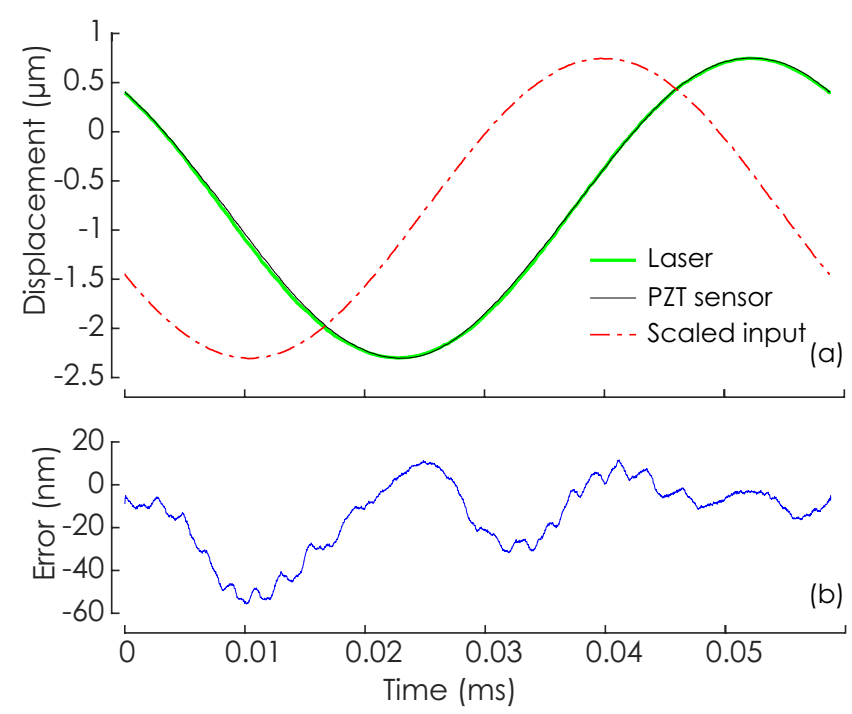

Fig. 17. Experimental response of the PZT sensor to a large signal sinusoidal excitation of $y$-axis at $17016 \mathrm{~Hz}$. The excitation voltage on the PZT actuator is $17 \mathrm{Vpp}$ and the calibration coefficient for the $\mathrm{PZT}$ sensor at this frequency is $-0.3407 \frac{\mu m}{\mathrm{~V}}$. responses have been found almost $180^{\circ}$ out of phase at each sinusoidal excitation in this high frequency range. Hence, they can be matched by a negative coefficient rather than the positive ones reported in Table II for the main sensing bandwidth. This coefficient, however, varies considerably with the frequency of the sinusoid, as indicated in Fig. 16.

Fig. 17 shows the time domain responses of the stage displacement and the scaled PZT sensor and actuator signals obtained at a frequency close to the dominant resonance of the $y$-axis, which is well above the frequency of the zeros. Note that the PZT sensor and stage displacement signals almost overlap and the error is very small. The resulting sensitivity of the PZT sensor at this frequency is also around $2.93 \frac{\mathrm{V}}{\mu \mathrm{m}}$, which is larger than those obtained in the sensing bandwidth.

\section{Resolution}

Resolution of an analog sensor is usually determined from the standard deviation of sensor noise [10], [55]. To characterize the sensor noise, the PZT sensor voltage of $y$ axis was directly recorded without any filter by a sampling rate of $50 \mathrm{kHz}$ while maintaining the $70 \mathrm{~V}$ bias voltage on the $y$-axis PZT actuator.
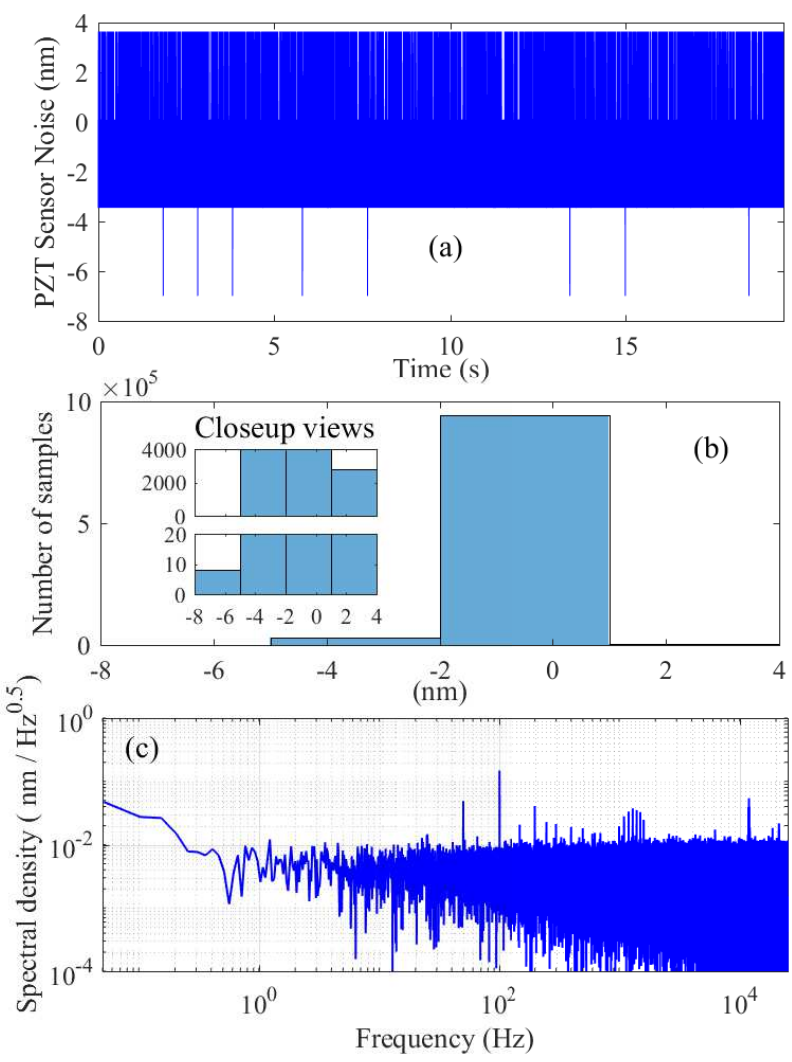

Fig. 18. (a) PZT sensor noise of $y$-axis in time-domain, (b) histogram, (c) spectral density.

Using the calibration coefficient reported in Table II, the resulting time-domain profile, histogram, and spectral density of the PZT sensor noise are as shown in Fig. 18. 
The time-domain data indicates that the sensor has a $3 \sigma$ resolution of $1.9 \mathrm{~nm}$, which is calculated from the $0.63 \mathrm{~nm}$ standard deviation of the noise in a $25 \mathrm{kHz}$ bandwidth. The spectral density of the PZT sensor has an average value of $3.5 \mathrm{pm} / \sqrt{\mathrm{Hz}}$, which is useful to approximate the sensor resolution within a desired bandwidth [23]. Hence, the $3 \sigma$-resolution of the PZT sensor with an effective bandwidth of $\Delta f \mathrm{~Hz}$ is around $10.5 \sqrt{\Delta f} \mathrm{pm}$.

\section{Conclusion}

Piezoelectric ceramics can be used for real-time measurement of time-varying displacement of nano-positioning stages. As the ceramic needs to experience a force proportional to the stage displacement, it has to be installed in series with the stage actuator. Additional parts are also required to properly insulating the PZT ceramic sensor from any actuator that requires high voltage values. The sensor exhibits minimum-phase zeros close to the imaginary axis at the resonance modes of the unloaded nano-positioner. Such zeros, which limit the sensor bandwidth, do not exist in the frequency response of the stage displacement when the positioner is preloaded by the actuators and the sensors. In addition, they are located at a frequency smaller than that of the dominant resonance mode of the loaded positioners. Hence, the upper corner frequency of the sensor bandwidth in the loaded positioner is below the dominant resonance mode of the unloaded positioners. The PZT sensor can also measure the stage displacement generated by single tone sinusoidal excitations at frequencies well above the zeros. Despite the low frequency range, the calibration factor in this high frequency range is negative and varies with the frequency considerably. The PZT sensor also exhibits a first-order high-pass response due to the electric capacitance property of the ceramic, which limits the sensing bandwidth at very low frequencies. However, it has many benefits, such as small noise level, small foot-print, high-frequency single-tone measurement, and no required signal conditioning instruments which makes them an ideal displacement sensor for time-varying applications such as scanning probe microscopy.

\section{ACKNOWLEDGMENT}

This work has been partially sponsored by the project PolyREM (Emergence Sorbonne Université), the French government research program Investissements d'avenir through the Robotex Equipment of Excellence (ANR-10EQPX-44), and the University of Newcastle Australia. We would like to thank Dr. Yuen K. Yong and Prof. Andrew J. Fleming for their valuable advices.

\section{REFERENCES}

[1] S. S. Aphale, A. J. Fleming, and S. O. R. Moheimani, "High speed nano-scale positioning using a piezoelectric tube actuator with active shunt control," IET Micro 85 Nano Letters, vol. 2, no. 1, pp. 9-12, 2007.

[2] S. Polit and J. Dong, "Development of a highbandwidth XY nanopositioning stage for high-rate Micro/Nanomanufacturing," IEEE/ASME Transactions on Mechatronics, vol. 16, pp. 724-733, Aug 2011.
[3] C.-X. Li, G.-Y. Gu, M.-J. Yang, and L.-M. Zhu, "Design, analysis and testing of a parallel-kinematic high-bandwidth XY nanopositioning stage," Review of Scientific Instruments, vol. 84, no. 12, p. 125111, 2013.

[4] A. San-Millan, V. Feliu-Batlle, and S. S. Aphale, "Application of a fractional order integral resonant control to increase the achievable bandwidth of a nanopositioner.," in 20th IFAC World Congress, vol. 50, pp. 14539 - 14544, 2017.

[5] M. Altaher and S. S. Aphale, "High-precision control of a piezodriven nanopositioner using fuzzy logic controllers," Computers, vol. 7, no. 1, p. Article No. 10, 2018.

[6] H. Huang, H. Zhao, Z. Yang, Z. Fan, S. Wan, C. Shi, and Z. Ma, "Design and analysis of a compact precision positioning platform integrating strain gauges and the piezoactuator," Sensors, vol. 12, pp. 9697-9710, July 2012.

[7] E. Guliyev, B. E. Volland, Y. Sarov, T. Ivanov, M. Klukowski, E. Manske, and I. W. Rangelow, "Quasi-monolithic integration of silicon-MEMS with piezoelectric actuators for high-speed non-contact atomic force microscopy," Measurement Science and Technology, vol. 23, no. 7, p. 074012, 2012.

[8] A. J. Fleming and K. K. Leang, "Integrated strain and force feedback for high performance control of piezoelectric actuators," Sensors and Actuators A, vol. 161, no. 1-2, pp. 256-265, 2010.

[9] Y. K. Yong and A. J. Fleming, "High-speed vertical positioning stage with integrated dual-sensor arrangement," Sensors and Actuators A: Physical, vol. 248, pp. 184 - 192, 2016.

[10] C. Lee and S. M. Salapaka, "Robust broadband nanopositioning: fundamental trade-offs, analysis, and design in a twodegree-of-freedom control framework," Nanotechnology, vol. 20, p. 035501, Dec. 2008.

[11] Y. K. Yong, S. O. R. Moheimani, B. J. Kenton, and K. K. Leang, "Invited review article: High-speed flexure-guided nanopositioning: Mechanical design and control issues," Review of Scientific Instruments, vol. 83, p. 121101, Dec. 2012.

[12] K. H. Koh, T. Kobayashi, J. Xie, A. Yu, and C. Lee, "Novel piezoelectric actuation mechanism for a gimbal-less mirror in $2 \mathrm{~d}$ raster scanning applications," Journal of Micromechanics and Microengineering, vol. 21, p. 075001, June 2011.

[13] T. Aimono, "Optical deflector including piezoelectric sensor incorporated into outermost piezoelectric cantilever," US Patent 13/846,028, Nov. 2013.

[14] Y. Park, S. Moon, J. Lee, K. Kim, S.-J. Lee, , and J.-H. Lee, "Gimbal-less two-axis electromagnetic microscanner with twist mechanism," Micromachines, vol. 9, p. 219, May 2018.

[15] K. An, S. Hong, S. Han, H. Lee, J. Yeo, and S. H. Ko, "Selective sintering of metal nanoparticle ink for maskless fabrication of an electrode micropattern using a spatially modulated laser beam by a digital micromirror device," ACS Applied Materials Es Interfaces, vol. 6, pp. 2786-2790, Feb. 2014. PMID: 24471931.

[16] K. Bakshi and A. V. Mulay, "A review on selective laser sintering: A rapid prototyping technology," IOSR Journal of Mechanical and Civil Engineering, 5th National Conference on Recent Developments in Mechanical Engineering [RDME-2016], vol. 04, pp. 53-57, Mar. 2016.

[17] W. Ameen, A. M. Ghaleb, M. Alatefi, H. Alkhalefah, and A. Alahmari, "An overview of selective laser sintering and melting research using bibliometric indicators," Virtual and Physical Prototyping, vol. 13, pp. 282-291, July 2018.

[18] https://www.npoint.com/npoint-products/.

[19] https://www.thorlabs.com/newgrouppage9.cfm?objectgroup_id =9862.

[20] https://www.thorlabs.com/newgrouppage9.cfm?objectgroup_id $=5360$.

[21] M. R. Nabavi and S. Nihtianov, "A survey of eddy current displacement sensors: Imperfections and signal conditioning methods," in 16th International Scientific and Applied Science Conference, Book 3: Electronics ET 2007, (Sozopol, BULGARIA), pp. 116-122, Sept. 2007. ISSN: 1313-1842.

[22] M. R. Nabavi and S. N. Nihtianov, "Design strategies for eddycurrent displacement sensor systems: Review and recommendations," IEEE Sensors Journal, vol. 12, pp. 3346-3355, Dec 2012.

[23] A. J. Fleming, "A review of nanometer resolution position sensors: operation and performance," Sensors and Actuators A: Physical, vol. 190, pp. 106-126, 2013.

[24] http://www.madcitylabs.com/picoq.html. 
[25] https://www.photonics.com/Articles/Piezoresistive_Sensors_ Facilitate_High-Speed/a16411.

[26] A. Furuta, M. Munekata, and T. Higuchi, "Precise positioning stage driven by multilayer piezo actuator using strain gauge," Japanese Journal of Applied Physics, vol. 41, pp. 6283-6286, Oct. 2002.

[27] P. Regtien and E. Dertien, "Ch. 8 - piezoelectric sensors," in Sensors for Mechatronics (Second Edition) (P. Regtien and E. Dertien, eds.), pp. 245 - 265, Elsevier, second ed., 2018.

[28] M. Vijaya, Piezoelectric Materials and Devices: Applications in Engineering and Medical Sciences. Taylor \& Francis, 2012.

[29] M. Chen-Glasser, P. Li, J. Ryu, and S. Hong, Piezoelectric Materials for Medical Applications, ch. 7, pp. 125-145. IntechOpen, 082018.

[30] https://www.americanpiezo.com/piezo-theory/pzt.html.

[31] M. Asadnia, A. G. P. Kottapalli, K. D. Karavitaki, M. E. Warkiani, J. Miao, D. P. Corey, and M. Triantafyllou, "From biological cilia to artificial flow sensors: Biomimetic soft polymer nanosensors with high sensing performance," Scientific Reports, vol. 6, p. 32955, Sept. 2016.

[32] H. Khan, A. Razmjou, M. Ebrahimi Warkiani, A. Kottapalli, and M. Asadnia, "Sensitive and flexible polymeric strain sensor for accurate human motion monitoring," Sensors, vol. 18, p. 418, Feb. 2018.

[33] D. H. Zeuch, S. T. Montgomery, and D. J. Holcomb, "Uniaxial compression experiments on lead zirconate titanate 95/5$2 \mathrm{Nb}$ ceramic: Evidence for an orientation-dependent, "maximum compressive stress" criterion for onset of the ferroelectric to antiferroelectric polymorphic transformation," Journal of Materials Research, vol. 15 , no. 3, pp. 689-703, 2000.

[34] D. Zhou, M. Kamlah, and D. Munz, "Uniaxial compressive stress dependence of the high-field dielectric and piezoelectric performance of soft PZT piezoceramics," Journal of Materials Research, vol. 19, p. 834âẨ̧842, Mar. 2004.

[35] Y. Tadesse, S. Priya, H. Stephanou, D. Popa, and D. Hanson, "Piezoelectric actuation and sensing for facial robotics," Ferroelectrics, vol. 345 , no. 1 , pp. 13-25, 2006.

[36] PCB PIEZOTRONICS, MTS SYSTEMS Corp., Reuseable Piezoelectric ICP Strain Sensor, Series 740.

[37] B. Yaghootkar, S. Azimi, and B. Bahreyni, "A high-performance piezoelectric vibration sensor," IEEE Sensors Journal, vol. 17, pp. 4005-4012, July 2017.

[38] A. Bazaei, M. Maroufi, and S. O. R. Moheimani, "Noise resilient 2-DoF washout tracking controller for nanopositioning systems subject to sensor drift," in 2015 American Control Conference (ACC), pp. 1199-1204, July 2015

[39] C. C. Collins and R. S. Mackay, "Zero frequency response from AC transducers," IRE Transactions on Medical Electronics, vol. ME-7, pp. 349-350, Oct 1960.

[40] A. N. Laskovski, S. O. R. Moheimani, and M. Yuce, "Piezoelectric strain voltage sensing at ultra low frequencies," Review of Scientific Instruments, vol. 82, p. 086113, Aug. 2011.

[41] A. N. Laskovski, M. R. Yuce, and S. R. Moheimani, "FMbased piezoelectric strain voltage sensor at ultra-low frequencies with wireless capability," Sensors and Actuators A: Physical, vol. 199 , pp. $49-55$, Sept. 2013.

[42] T. Kleckers, "Force sensors based on strain gages and piezoelectric crystal-based force transducers in mechatronic systems-a comparison," in SENSOR+TEST Conferences 2011, Mechanical Sensors I, (Nurnberg, Germany), pp. 257-260, June 2011.

[43] T. Kleckers, "Force sensors for strain gauge and piezoelectric crystal-based mechatronic systems-a comparison," in IEEE International Instrumentation and Measurement Technology Conference Proceedings, pp. 2306-2308, May 2012.

[44] https://www.hbm.com/en/3719/piezoelectric-or-strain-gaugebased-force-transducers/.

[45] M. Asadnia, A. G. P. Kottapalli, Z. Shen, J. Miao, and M. Triantafyllou, "Flexible and surface-mountable piezoelectric sensor arrays for underwater sensing in marine vehicles," IEEE Sensors Journal, vol. 13, pp. 3918-3925, Oct. 2013.

[46] A. M. Hamed, Z. T. H. Tse, I. Young, B. L. Davies, and M. Lamperth, "Applying tactile sensing with piezoelectric materials for minimally invasive surgery and magnetic-resonance-guided interventions," Proceedings of the Institution of Mechanical Engineers, Part H: Journal of Engineering in Medicine, vol. 223, no. 1 , pp. 99-110, 2009.
[47] R. J. Miller, "Artificial skin tactile sensor for prosthetic and robotic applications," Master thesis, California Polytechnic State University, San Luis Obispo, Dec. 2010.

[48] D. P. J. Cotton, P. H. Chappell, A. Cranny, N. M. White, and S. P. Beeby, "A novel thick-film piezoelectric slip sensor for a prosthetic hand," IEEE Sensors Journal, vol. 7, pp. 752-761, May 2007

[49] M. Rossi, M. Nardello, L. Lorenzelli, and D. Brunelli, "Dual mode pressure sensing for lower-limb prosthetic interface," in Eurosensors 2017, vol. 1, (Paris, France), p. 593, MDPI, Sept. 2017.

[50] M. Rossi, M. Nardello, L. Lorenzelli, and D. Brunelli, "Dual mode pressure sensing for prosthetic interfaces," in 7th IEEE International Workshop on Advances in Sensors and Interfaces (IWASI), pp. 109-114, June 2017.

[51] R. Knobel and A. N. Cleland, "Piezoelectric displacement sensing with a single-electron transistor," Applied Physics Letters, vol. 81, no. 12 , pp. 2258-2260, 2002.

[52] J. Li, J. T. Santos, and M. A. Sillanpää, "High-precision displacement sensing of monolithic piezoelectric disk resonators using a single-electron transistor," Journal of Low Temperature Physics, vol. 191, pp. 316-329, Jun 2018.

[53] M. Umapathy, Y. Sujan, and K. Suresh, "Piezoelectric based resonance displacement sensor," in 2013 IEEE Sensors Applications Symposium Proceedings, pp. 193-196, Feb 2013.

[54] https://www.thorlabs.com/navigation.cfm?guide_id=82.

[55] https://www.sensorsmag.com/embedded/noise-measurement.

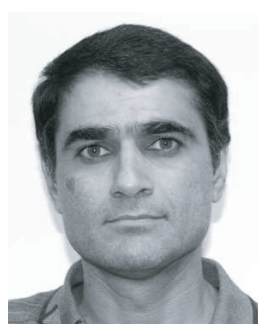

Ali Bazaei (M'10) received B.Sc. and M.Sc. degrees from Shiraz University and a Ph.D. degree from Western University, all in electrical engineering in 1992, 1995, and 2009, respectively. Since 2009, he held postdoctoral research fellowship, research associate, and higher degree research positions with University of Newcastle, Australia and with Institute for Intelligent Systems and Robotics (ISIR) at Sorbonne University. His research interests include the general area of nonlinear systems including control and modeling of structurally flexible systems, friction modeling and compensation, neural networks, and micro-position sensors, and Nano-positioning in Scanning Probe Microscopy.

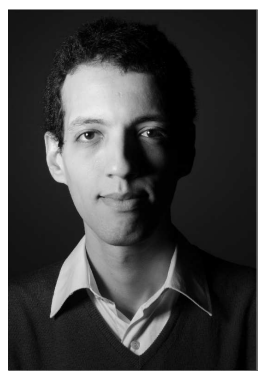

Mokrane Boudaoud received the engineering degree in automatic control from the University of science and technology Houari Boumédiène (USTHB, Algiers, Algeria), and a MS degree in mechatronics and microrobotics at the University of Franche-Comté (UFC, Besançon, France) in 2009. He received in 2012 the $\mathrm{PhD}$ degree in control engineering at AS2M (Automatic Control and MicroMechatronic Systems) department of Femto-st Institute. He is currently Associate Professor at University Pierre et Marie Curie / Institut des Systèmes Intelligents et de Robotique. His field of interest includes modeling and robust control of multi-degree-of-freedom nano-robotic systems operating under a scanning electron microscope (SEM). Dr. Boudaoud is an Associate Editor of the journal Mechatronics (Elsevier). 


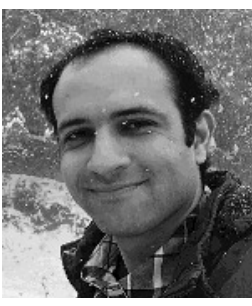

Massoud Hemmasian Ettefagh received his B.Sc. degree from Isfahan University of Technology, Iran in 2008, and M.Sc. and P.h.D. degrees from Amirkabir University of Technology, Iran in 2011 and 2018, all in mechanical engineering. During 2016-2019 he was a visiting fellow at University of Newcastle, Australia and Universidad Carlos III de Madrid, Spain. Currently, he is with the Huazhong University of Science and Technology (HUST), China as a postdoctoral fellowship. His research interests include real-time control, constrained control, model predictive control, time-varying systems, differential geometric methods in nonlinear systems, and design and control of nanopositioning platforms for AFMs.

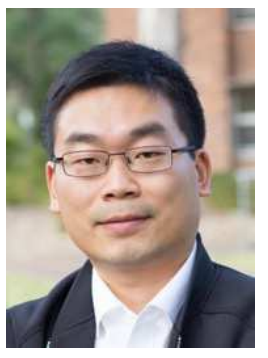

Zhiyong CHEN received the B.E. degree from the University of Science and Technology of China, and the M.Phil. and Ph.D. degrees from the Chinese University of Hong Kong, in 2000, 2002 and 2005, respectively. He worked as a research associate at the University of Virginia during 2005-2006. He joined the University of Newcastle, Australia, in 2006, where he is currently a professor. He is also a Changjiang chair professor with Central South University, Changsha, China. His research interests include non-linear systems and control, biological systems, and multi-agent systems. He is/was an associate editor of Automatica, IEEE Transactions on Automatic Control and IEEE Transactions on Cybernetics.

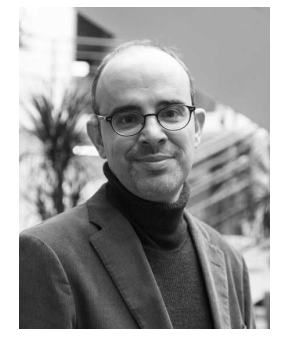

Stéphane Régnier received his $\mathrm{PhD}$ degree in Mechanics and Robotics from the University of Pierre and Marie Curie, Paris, France in 1996. He is currently Professor at the Institute of Intelligent Systems and Robotics (ISIR), University of Pierre and Marie Curie, Paris, France. He has been head of the ISIR micromanipulation team since 2001. His research interests are focused on micro and nano manipulation, teleoperation and haptic feedback logical cell characterization. at the nanoscale, micromechatronics and bio- 